\title{
A new approach to Color Edge Detection
}

\author{
Pablo A. Flores-Vidal ${ }^{a}$ and Daniel Gómez ${ }^{b}$ and Javier Castro ${ }^{b}$ and Guillermo Villarino ${ }^{b}$ and Javier Montero $^{a}$ \\ ${ }^{a}$ Statistics and Operational Research, Faculty of Mathematics, Complutense university, \\ Plaza de las Ciencias 3, pflores@ucm.es \\ ${ }^{b}$ Statistics and Data Science, Faculty of Statistical Studies, Complutense university, \\ Avda. Puerta de Hierro s/n, dagomez@estad.ucm.es
}

\begin{abstract}
Most edge detection algorithms deal only with grayscale images, and the way of adapting them to use with RGB images is an open problem. In this work, we explore different ways of aggregating the color information of a RGB image for edges extraction, and this is made by means of well-known edge detection algorithms. In this research, it is been used the set of images from Berkeley. In order to evaluate the algorithm's performance, $\mathrm{F}$ measure is computed. The way that color information -the different channels- is aggregated is proved to be relevant for the edge detection task. Moreover, post-aggregation of channels performed significatively better than the classic approach (pre-aggregation of channels).
\end{abstract}

Keywords: Color edge detection, Multichannel edge detection, RGB, Preaggregation, Post-aggregation, Crispy aggregation, Fuzzy aggregation.

\section{Introduction}

RGB images for image processing have not been employed so often compared with grayscale images. This is specially true in the case of the edge detection problem, where dealing with color images introduces some complications.

The RGB images are built in the RGB space color. This space color it is based in human perception as human vision has three different cone cells, one captures the red luminosity, meanwhile the other two do the same with green and blue luminosity respectively. Human vision employes rods, a second kind of cell, but this one only can process intensity not color [1]. Due to this, "Three numerical components are necessary and sufficient to describe a color..." as it is said by Bogumil [1]. In this sense, the RGB color space should be ideal for retaining all the color information, as it is using three components for it (Red, Green and Blue).

Marr [16] pointed out that color could be relevant due that it "carries information that often has important biological significance". This information could help to distinguish "wheter a fruit is ripe, wheter a leaf is green and supple, wheter an insect is likely to be poisonous, and many other things".

As we will see in the comparatives, the edge detection using RGB channels performs better than just using grayscale edges (see Section 4 ).

The remaining of this paper is organized as follows: The next section 2 is dedicated to some preliminaries in edge detection problems, including the case of color edge detection. The different approaches for aggregating the color channels are presented in section 3 . Finally, in the section 4 and section 5 we present some comparative results and conclusions respectively.

\section{Preliminaries}

In this section are introduced some needed concepts of image processing and edge extraction problem. Let us denote by $I$ a digital image, and by $(i, j)$ the pixel coordinates of the spatial domain. For simplification purposes the coordinates are integers, where each point $(i, j)$ represents a pixel with $i=\ldots, n$ and $j=\ldots, m$. Therefore, the size of an image, $n \times m$, is the number of its horizontal pixels multiplied by its number of verticals. As we are dealing with color images, then a $k=1, \ldots, \tilde{k}$ index is needed for expressing the number of channels in the image. Thus, let us denote by $\mathrm{I}=P_{i, j}^{k}$ the spectral information associated with each pixel $(\mathrm{i}, \mathrm{j})$ at channel $k$. As well, $I=P_{i, j}^{k}$ is equivalent to $\tilde{k}$ images of one single channel (grayscale images) $I=\left\{I^{1}, \ldots, I^{\tilde{k}}\right\}$. The values range of this information depends on the type of image considered. 
- binary map: $I_{b i n}=P_{i, j} \in\{.255\}$ (as well it is usually expressed as $\{.1\}$ ).

- grayscale image: $I_{\text {gray }}=P_{i, j} \in\{.1, \ldots, 255\}$.

- $R G B$ image: $I_{R G B}=P_{i, j} \in\{.1, \ldots, 255\}^{3}$. $(\mathrm{R}=$ Red $; \mathrm{G}=$ Green and $\mathrm{B}=$ Blue $)$.

- soft image: $I_{\text {soft }}=P_{i, j} \in[.1]$. As well it is referred as a normalized grayscale image.

Edge detection technique has not a single acepted definition [13]. The most common is to consider it as a technique that identifies the significant luminosity changes in the image.

The concept of edge detection in color images is more advanced than in grayscale edge detection, and this makes it much more complex to deal with. Now, the concept of color similarity becomes crucial. Different approaches to deal with color edge detection have been proposed:

(I) Individual channel: The edge detection is computed for each single channel. This is the approach employed in this paper.

(II) Vector-based approach: An aggregation function is applied, such a median filter [20], a range operator [22], or other statistical aggregation methods [6].

Other authors $([18,5])$ have considered as a third important approach the one of working with gradients that result of combining from different colors, but we consider that this approach can be included in the vector-based approach and this simplifies the taxonomy of color edge detection. The interesting approach developed in [18] which is based on computing distributions of colors of two hypothesized regions could be considered as a different one, but we did not find many other authors in the literature following this aproach. As well, in this paper we have focused in the specific case of RGB images instead of dealing with multispectral or hyperspectral imagery as it is more common in remote sensing field [15].

From a mathematical point of view an edge detection algorithm is a function that converts a digital image into a binary image. We would like to emphasize that most of edge detection algorithms only deal with grayscale images, meanwhile there are a high number of segmentation algorithms dealing with color images $[12,11]$. From this idea, it is clear that an edge detection algorithm transforms an image into a binary image or $I_{b i n}$. In this binary image, the white pixels (the pixels where $P_{i, j}=1$ ) are those that have been identify by the edge detection algorithm as edge pixels. In the case of $I_{b i n}$ being the output of an edge detection algorithm we call it $I_{\text {solut }}$, as this image is the solution of the algorithm.

\section{$3 \quad$ Aggregating channels in color edge detection}

Although there exist some approaches (vector-based approaches) that detect edges in color images based on dissimilarity/distance measures between colors, in this work, we have focused on individual channel approach. We have chosen this approach instead of vector-based since it is easy to find situations in which any distance measure tends to compesate significant differences in specific channels. Contrarely, with the individual channel aproach this limitation related to these compesations can be easily avoid. All the aggregation methodologies are applied after the operator's gradient is computed -as well known as blending-aggregation phase [9]- for different directions. We are aware of the possibilities of starting our study considering the different directions -horizontal and vertical- of change in the spectral information prior to their gradient aggregation, but we decided to start this research after the blending-aggregation phase for simplification purposes.

(a) Crispy pre-aggregation (Methodology A): Aggregating the intensity components in one single gray channel. This means that first we aggregate with simple addition the different color channels into one single gray channel and then an edge detection algorithm is applied over the resulted grayscale image. This can be seen as the classic method, as it is the most common procedure employed in the literature. When dealing with RGB images we have three channels $(\tilde{\mathrm{k}}=3) I_{R G B}^{1}$, $I_{R G B}^{2}$ and $I_{R G B}^{3}$ corresponding to red, green and blue channels respectively.

$I_{\text {gray }}=\frac{I_{R G B}^{1}+I_{R G B}^{2}+I_{R G B}^{3}}{3}$

As we could be dealing with more channels, a more general formula can be written:

$I_{\text {gray }}=\frac{I^{1}+\ldots+I^{k}}{\ddot{k}}$

As well, it has been used the weighted addition with interesting results [6]. This approach allows giving different importance to each color channel.

Once we have a single grayscale channel, an algorithm's operator is applied following standard procedures: $I_{\text {soft }}=\operatorname{edge}\left(I_{\text {gray }}\right)$ as a general formula, and the specific cases employed in this paper being 
$I_{\text {soft }}=\operatorname{Sobel}\left(I_{\text {gray }}\right)$ and $I_{\text {soft }}=\operatorname{Canny}\left(I_{\text {gray }}\right)$. After the operator the thinning -non maximum suppression- is applied. As the final step, over the thinned version of the soft image the binarized image $I_{\text {solut }}$ is produced. It results from the soft image after a threshold value is applied (for a detailed explanation of the different steps in edge detection techniques see $[9,8,7]): I_{\text {solut }}=\operatorname{threshold}\left(I_{\text {soft }}\right)$ where threshold() means that a threshold function is applied over the thinned version of the soft image. An scheme of the three methodologies is summarized in Figure 1.

(b) Crispy post-aggregation (Methodology B): Applying an edge detection operator over each channel separately, which produces $\tilde{K}$ different edges maps. Then, all the $\tilde{\mathrm{K}}$ resulted binarized images will be aggregated into a single one. We can see in Algorithm 1 this methodology:

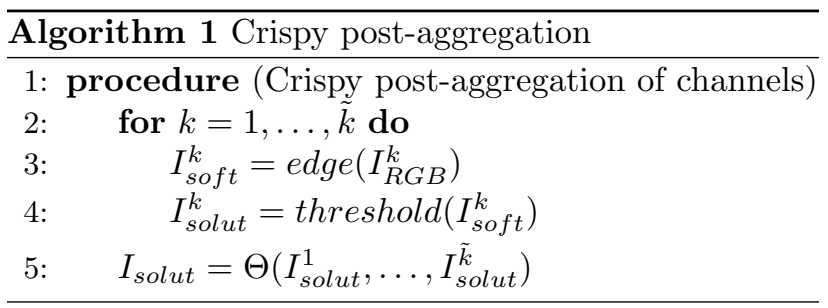

(c) Fuzzy post-aggregation (Methodology C): In this case the aggregation function is applied not over the already binarized image but over the soft image corresponding to each color channel. This soft image is made of what we consider as candidates to be edge pixels. The binarized image is produced at the last step of the algorithm, following a soft approach that we have called "fuzzy" approach:

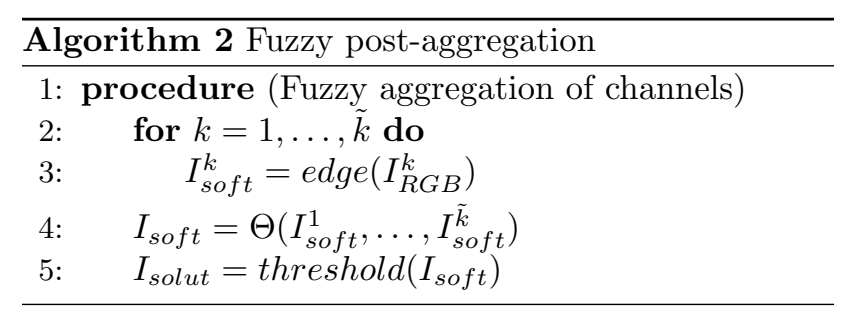

We have used different aggregation functions $\Theta()$ :

(I) The sum: $I_{\text {solut }}=\sum_{k=1}^{\tilde{k}} I_{\text {solut }}^{k}$.

(II) The mean: $I_{\text {solut }}=\frac{\sum_{k=1}^{\tilde{k}} I_{\text {solut }}^{k}}{n}$.

(III) The maximum: $I_{\text {solut }}=\max \left(I_{\text {solut }}^{1}, \ldots, I_{\text {solut }}^{\tilde{k}}\right)$.

\section{Comparatives and results}

The well-known algorithms of Sobel [19] and Canny [2] have been employed. In the case of Sobel's we have used the standard version of it. By contrast, for simplification purposes the Canny's version employed has a Gaussian filter size of $5 \times 5$ pixels and $\sigma=2$, which is enough for a good quality in the edge extraction process. Both algorithms have been tested in the three different methodologies -A, B and C- explained in Section 3. C method has been applied with two different aggregation function, mean (C1) and maximum (C2), meanwhile B method employed only the maximum. In Table 1 we see the maximum $\mathrm{F}$ results (corresponding to the human with the highest $\mathrm{F}$-measure values for each image) after applying the different versions of Sobel algorithm -for each methodology- for the first 50 images of the training dataset of Berkeley [17]. The automated threshold value is written next to each $\mathrm{F}$ value. The same is showed for Canny's in Table 2 where we used the low threshold value as a $40 \%$ of the high threshold value as we have often employed often this percentage after testing with the whole Berkeley set of images and verified that performs well enough.

\subsection{Statistical analysis}

This section is aimed to assess the improvements and differences achieved by the methods proposed in this paper. To do so, we use some hypothesis validation techniques in order to give statistical support to the analysis of the results.

Specifically, the Wilcoxon rank test [21] is employed as a non-parametric statistical procedure for making pairwise comparisons between two algorithms. For multiple comparisons, we use the Friedman aligned ranks test, which is recommended in the literature $[4,10]$ to detect statistical differences among a group of results by considering different levels of confidence for each method. Finally, the Holm post-hoc test [14] is performed to find which algorithms reject the equality hypothesis with respect to a selected control method.

The Wilcoxon test for the Sobel's case, which is showed in Table 3, can be interpreted in different ways. Firstly, it is possible to asses whether the postaggregation approach outperforms the pre-aggregation approach by comparing methods B, C1 and C2 with respect to $\mathrm{A}$. And effectively they do it (with a low p-value).

Going beyond, we contrasted the hypothesis of equality between the results reached by each fuzzy based method (C1 and $\mathrm{C} 2$ ) and each crispy one (A and B) and rejected it for each possible combination with a p-value of exactly 0 . 


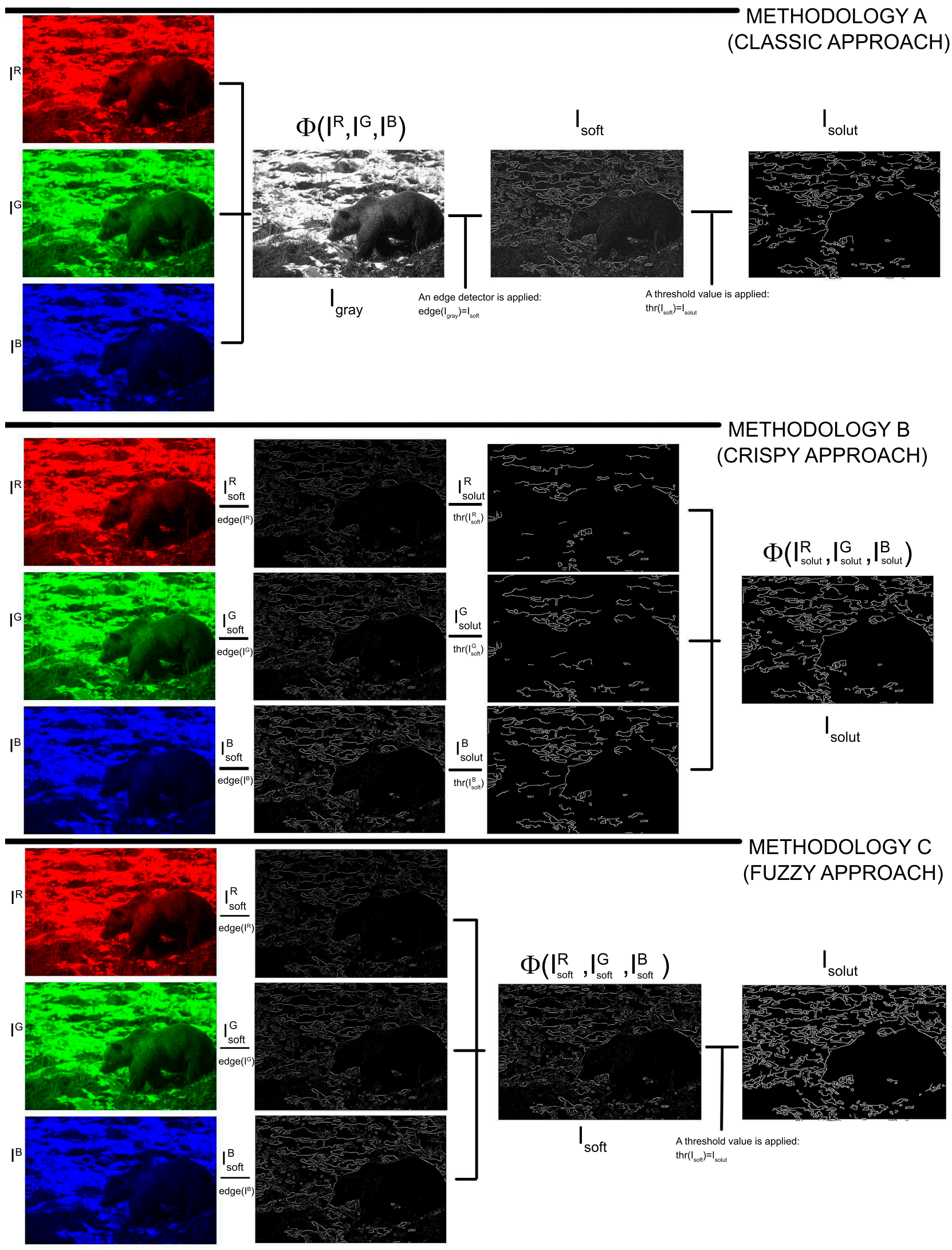

Figure 1: Scheme of methodologies A, B and C. 


\begin{tabular}{|c|c|c|c|c|c|c|c|c|}
\hline \multirow[b]{2}{*}{ Image } & \multicolumn{2}{|c|}{$\begin{array}{l}\text { Sobel } \\
\text { Gray } \\
\text { (Method } \\
\text { A) }\end{array}$} & \multicolumn{2}{|c|}{$\begin{array}{l}\text { Sobel RGB } \\
\text { Crispy } \\
\text { Maximum } \\
\text { Aggre- } \\
\text { gation } \\
\text { (Method } \\
\text { B) }\end{array}$} & \multicolumn{2}{|c|}{$\begin{array}{l}\text { Sobel } \\
\text { RGB } \\
\text { Fuzzy } \\
\text { Mean Ag- } \\
\text { gregation } \\
\text { (Method } \\
\text { C1) }\end{array}$} & \multicolumn{2}{|c|}{$\begin{array}{l}\text { Sobel } \\
\text { RGB } \\
\text { Fuzzy } \\
\text { Maximum- } \\
\text { Aggregation } \\
\text { (Method } \\
\text { C2) }\end{array}$} \\
\hline & Fmax & Thr\% & Fmax & Thr\% & Fmax & Thr\% & Fmax & Thr\% \\
\hline 100075 & .371 & 27 & .407 & 31 & .421 & 27 & .454 & 29 \\
\hline 100080 & .304 & 24 & .312 & 26 & .383 & 22 & .382 & 23 \\
\hline 100098 & .507 & 50 & .508 & 54 & .693 & 45 & .698 & 50 \\
\hline 103041 & .372 & 25 & .375 & 24 & .555 & 21 & .558 & 24 \\
\hline 104022 & .535 & 25 & .535 & 29 & .591 & 22 & .595 & 24 \\
\hline 105019 & .649 & 28 & .646 & 31 & .656 & 29 & .651 & 31 \\
\hline 105053 & .431 & 21 & .430 & 23 & .458 & 20 & .450 & 23 \\
\hline 106020 & .194 & 15 & .188 & 16 & .253 & 22 & .243 & 20 \\
\hline 106025 & .515 & 27 & .541 & 27 & .647 & 27 & .674 & 27 \\
\hline 108041 & .222 & 29 & .214 & 30 & .286 & 22 & .277 & 22 \\
\hline 108073 & .303 & 54 & .290 & 45 & .382 & 24 & .363 & 42 \\
\hline 109034 & .171 & 36 & .196 & 63 & .217 & 27 & .223 & 66 \\
\hline 112082 & .357 & 43 & .344 & 51 & .385 & 42 & .373 & 47 \\
\hline 113009 & .632 & 19 & .623 & 20 & .733 & 18 & .722 & 21 \\
\hline 113016 & .480 & 44 & .482 & 55 & .512 & 44 & .515 & 57 \\
\hline 113044 & .451 & 50 & .483 & 55 & .467 & 48 & .498 & 57 \\
\hline 117054 & .372 & 43 & .373 & 47 & .468 & 41 & .469 & 41 \\
\hline 118020 & .311 & 33 & .323 & 37 & .433 & 24 & .442 & 31 \\
\hline 118035 & .649 & 53 & .709 & 56 & .774 & 28 & .799 & 58 \\
\hline 12003 & .350 & 34 & .375 & 42 & .544 & 28 & .554 & 32 \\
\hline 12074 & .402 & 36 & .382 & 38 & .574 & 38 & .544 & 45 \\
\hline 122048 & .352 & 41 & .341 & 43 & .479 & 29 & .472 & 29 \\
\hline 124084 & .518 & 21 & .580 & 39 & .631 & 18 & .665 & 30 \\
\hline 126039 & .509 & 21 & .524 & 23 & .595 & 19 & .607 & 22 \\
\hline 130034 & .208 & 44 & .207 & 47 & .227 & 40 & .234 & 42 \\
\hline 134008 & .206 & 22 & .202 & 22 & .243 & 23 & .239 & 24 \\
\hline 134052 & .374 & 51 & .366 & 55 & .449 & 57 & .442 & 56 \\
\hline 135037 & .387 & 38 & .379 & 41 & .748 & 13 & .728 & 19 \\
\hline 135069 & .910 & 40 & .908 & 53 & .945 & 32 & .943 & 41 \\
\hline 138032 & .188 & 41 & .189 & 48 & .317 & 28 & .312 & 31 \\
\hline 138078 & .569 & 45 & .603 & 45 & .694 & 28 & .692 & 29 \\
\hline 140055 & .227 & 20 & .280 & 22 & .278 & 21 & .331 & 21 \\
\hline 140075 & .431 & 39 & .459 & 46 & .535 & 22 & .547 & 30 \\
\hline 144067 & .233 & 41 & .238 & 43 & .263 & 41 & .265 & 42 \\
\hline 145014 & .357 & 43 & .374 & 50 & .502 & 31 & .515 & 36 \\
\hline 145053 & .351 & 31 & .353 & 31 & .412 & 17 & .415 & 27 \\
\hline 147021 & .341 & 42 & .343 & 43 & .396 & 32 & .401 & 37 \\
\hline 147062 & .385 & 62 & .386 & 64 & .465 & 59 & .466 & 63 \\
\hline 15004 & .297 & 38 & .309 & 40 & .376 & 40 & .387 & 40 \\
\hline 15088 & .542 & 55 & .545 & 56 & .569 & 55 & .569 & 56 \\
\hline 151087 & .608 & 40 & .621 & 45 & .639 & 42 & .648 & 45 \\
\hline 153077 & .437 & 25 & .444 & 26 & .503 & 23 & .517 & 23 \\
\hline 153093 & .443 & 20 & .457 & 24 & .593 & 19 & .602 & 24 \\
\hline 155060 & .493 & 37 & .505 & 36 & .570 & 38 & .594 & 36 \\
\hline 156079 & .465 & 32 & .463 & 42 & .505 & 36 & .505 & 44 \\
\hline 157036 & .449 & 46 & .447 & 50 & .703 & 48 & .702 & 55 \\
\hline 159029 & .205 & 43 & .213 & 46 & .305 & 42 & .315 & 44 \\
\hline 159045 & .449 & 35 & .457 & 35 & .514 & 30 & .528 & 34 \\
\hline 159091 & .478 & 42 & .483 & 46 & .534 & 42 & .540 & 25 \\
\hline 16052 & .208 & 21 & .203 & 25 & .445 & 20 & .431 & 48 \\
\hline Min & .171 & 15 & .188 & 16 & .217 & 13 & .223 & 19 \\
\hline Mean & .404 & 36 & .412 & 40 & .497 & 31 & .502 & 36 \\
\hline Max & .910 & 62 & .908 & 64 & .945 & 59 & .943 & 66 \\
\hline
\end{tabular}

Table 1: Aggregating color channels using Sobel algorithm 


\begin{tabular}{|c|c|c|c|c|c|c|c|c|}
\hline \multirow[b]{2}{*}{ Image } & $\begin{array}{l}\text { Canny } \\
\text { Gray } \\
\text { Channel } \\
\text { (Method } \\
\text { A) }\end{array}$ & & $\begin{array}{l}\text { Canny } \\
\text { RGB } \\
\text { Crispy } \\
\text { Max } \\
\text { gregati } \\
\text { (Metho } \\
\text { B) }\end{array}$ & & $\begin{array}{l}\text { Canny } \\
\text { RGB } \\
\text { Fuzzy } \\
\text { Mean- } \\
\text { Aggreg } \\
\text { (Metho } \\
\text { C1) }\end{array}$ & tion & $\begin{array}{l}\text { Canny } \\
\text { RGB } \\
\text { Fuzzy } \\
\text { Maxim } \\
\text { Aggreg } \\
\text { (Metho } \\
\text { C2) }\end{array}$ & $\begin{array}{l}\text { m- } \\
\text { tion }\end{array}$ \\
\hline & Fmax & Thr\% & Fmax & Thr\% & Fmax & Thr\% & Fmax & Thr\% \\
\hline 100075 & .438 & 42 & .469 & 47 & .436 & 38 & .473 & 52 \\
\hline 100080 & .389 & 24 & .386 & 27 & .389 & 24 & .391 & 27 \\
\hline 100098 & .718 & 75 & .713 & 80 & .713 & 69 & .691 & 83 \\
\hline 103041 & .555 & 30 & .545 & 34 & .538 & 29 & .533 & 34 \\
\hline 104022 & .630 & 31 & .621 & 35 & .614 & 30 & .610 & 44 \\
\hline 105019 & .728 & 37 & .726 & 40 & .715 & 29 & .711 & 40 \\
\hline 105053 & .491 & 29 & .498 & 38 & .504 & 25 & .485 & 41 \\
\hline 106020 & .270 & 29 & .255 & 29 & .260 & 23 & .252 & 30 \\
\hline 106025 & .719 & 34 & .733 & 43 & .699 & 34 & .729 & 47 \\
\hline 108041 & .290 & 30 & .283 & 41 & .283 & 40 & .279 & 41 \\
\hline 108073 & .389 & 36 & .375 & 37 & .394 & 35 & .370 & 60 \\
\hline 109034 & .282 & 88 & .328 & 90 & .260 & 40 & .341 & 88 \\
\hline 112082 & .432 & 52 & .388 & 53 & .431 & 50 & .373 & 53 \\
\hline 113009 & .733 & 24 & .728 & 29 & .704 & 23 & .711 & 38 \\
\hline 113016 & .741 & 49 & .714 & 76 & .711 & 49 & .700 & 78 \\
\hline 113044 & .578 & 61 & .635 & 68 & .556 & 56 & .626 & 70 \\
\hline 117054 & .506 & 54 & .504 & 66 & .497 & 65 & .501 & 66 \\
\hline 118020 & .454 & 53 & .468 & 60 & .446 & 32 & .467 & 60 \\
\hline 118035 & .793 & 48 & .800 & 67 & .789 & 44 & .789 & 67 \\
\hline 12003 & .597 & 46 & .585 & 61 & .591 & 43 & .572 & 68 \\
\hline 12074 & .592 & 89 & .550 & 89 & .586 & 61 & .512 & 83 \\
\hline 122048 & .530 & 74 & .518 & 80 & .500 & 61 & .519 & 81 \\
\hline 124084 & .621 & 27 & .706 & 48 & .648 & 24 & .674 & 47 \\
\hline 126039 & .601 & 30 & .624 & 37 & .594 & 22 & .616 & 35 \\
\hline 130034 & .321 & 65 & .337 & 74 & .294 & 54 & .343 & 74 \\
\hline 134008 & .296 & 39 & .286 & 43 & .280 & 33 & .285 & 43 \\
\hline 134052 & .524 & 61 & .529 & 68 & .499 & 58 & .509 & 72 \\
\hline 135037 & .742 & 47 & .722 & 47 & .711 & 29 & .713 & 47 \\
\hline 135069 & .934 & 61 & .933 & 78 & .931 & 53 & .916 & 80 \\
\hline 138032 & .346 & 78 & .345 & 79 & .354 & 57 & .341 & 79 \\
\hline 138078 & .766 & 50 & .785 & 54 & .721 & 50 & .789 & 60 \\
\hline 140055 & .309 & 27 & .399 & 34 & .319 & 26 & .389 & 38 \\
\hline 140075 & .595 & 47 & .647 & 54 & .590 & 36 & .642 & 54 \\
\hline 144067 & .321 & 80 & .328 & 75 & .320 & 77 & .333 & 75 \\
\hline 145014 & .501 & 80 & .520 & 86 & .497 & 36 & .523 & 87 \\
\hline 145053 & .515 & 48 & .527 & 61 & .531 & 48 & .510 & 61 \\
\hline 147021 & .451 & 42 & .433 & 42 & .449 & 51 & .438 & 44 \\
\hline 147062 & .527 & 67 & .526 & 74 & .510 & 63 & .508 & 72 \\
\hline 15004 & .459 & 57 & .482 & 65 & .438 & 55 & .472 & 65 \\
\hline 15088 & .553 & 68 & .537 & 73 & .536 & 68 & .569 & 78 \\
\hline 151087 & .736 & 49 & .732 & 55 & .707 & 43 & .745 & 55 \\
\hline 153077 & .532 & 45 & .547 & 52 & .533 & 39 & .535 & 57 \\
\hline 153093 & .651 & 44 & .668 & 50 & .635 & 38 & .678 & 50 \\
\hline 155060 & .634 & 54 & .645 & 58 & .619 & 55 & .643 & 56 \\
\hline 156079 & .558 & 46 & .557 & 59 & .549 & 41 & .545 & 53 \\
\hline 157036 & .806 & 71 & .814 & 75 & .796 & 64 & .814 & 75 \\
\hline 159029 & .383 & 63 & .391 & 70 & .401 & 66 & .384 & 69 \\
\hline 159045 & .481 & 50 & .504 & 52 & .488 & 36 & .504 & 56 \\
\hline 159091 & .629 & 66 & .648 & 77 & .613 & 58 & .623 & 78 \\
\hline 16052 & .456 & 50 & .437 & 54 & .456 & 40 & .426 & 55 \\
\hline $1 \mathrm{NIII}$ & .270 & 24 & .255 & 27 & .260 & 22 & .252 & 27 \\
\hline Mean & .542 & 51 & .549 & 58 & .533 & 44 & .542 & 59 \\
\hline Max & .934 & 89 & .933 & 90 & .931 & 77 & .916 & 88 \\
\hline
\end{tabular}

Table 2: Aggregating color channels using Canny algorithm 


\begin{tabular}{cccc} 
Comparison & $R^{+}$ & $R^{-}$ & p-val \\
\hline Post-aggregation vs. pre-aggregation & & \\
\hline Method B vs. Method A & 932.0 & 343.0 & $\mathbf{0 . 0 0 4 4}$ \\
Method C1 vs. Method A & 1275.0 & 0.0 & $\mathbf{0}$ \\
Method C2 vs. Method A & 1275.0 & 0.0 & $\mathbf{0}$ \\
Fuzzy vs. Crispy & & & \\
\hline Method C2 vs. Method B & 1275.0 & 0.0 & $\mathbf{0}$ \\
Method C1 vs. Method B & 1270.0 & 5.0 & $\mathbf{0}$ \\
\hline Between fuzzy aggregations & & & \\
Method C2 vs. Method C1 & 843.0 & 432.0 & $\mathbf{0 . 0 4 6 7}$ \\
\hline
\end{tabular}

Table 3: Wilcoxon Test to compare the different aggregation methods for the Sobel's algorithm.

\begin{tabular}{cccc} 
Comparison & $R^{+}$ & $R^{-}$ & $\mathrm{p}$-val \\
\hline RGB vs. Grey Scale & & & \\
\hline Method B vs. Method A & 778.0 & 497.0 & 0.173478 \\
Method A vs. Method C1 & 1061.0 & 214.0 & $\mathbf{0 . 0 0 0 0 4 3}$ \\
Method A vs. Method C2 & 676.0 & 599.0 & 0.706562 \\
Fuzzy vs. Crispy & & & \\
\hline Method B vs. Method C1 & 1033.0 & 242.0 & $\mathbf{0 . 0 0 0 1 3 2}$ \\
Method B vs. Method C2 & 1021.0 & 254.0 & $\mathbf{0 . 0 0 0 2 1}$ \\
\hline Between fuzzy aggregations & & & \\
Method C2 vs. Method C1 & 837.0 & 438.0 & 0.053526 \\
\hline
\end{tabular}

Table 4: Wilcoxon Test to compare the different aggregation methods for the Canny's algorithm.

In the Canny's case, which is showed in Table 4, both Method A and B outperformed "fuzzy" methods (C1 and C2). This results are commented in Section 5 .

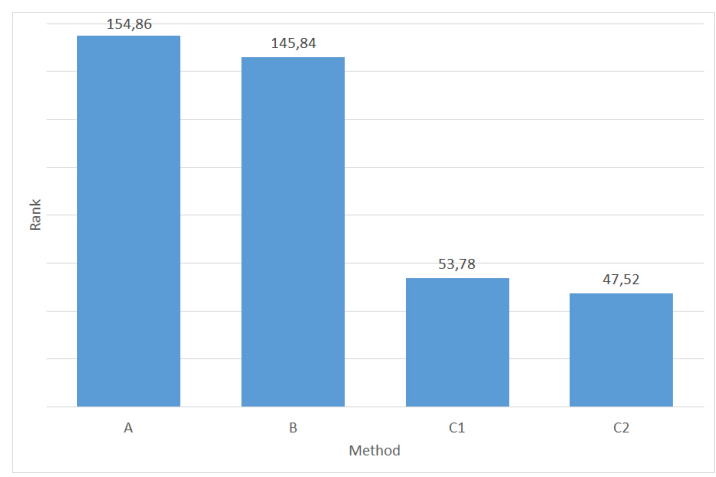

Figure 2: Rankings of the three compared methods employing Sobel's algorithm.

Finally, inside the soft ("fuzzy") frame, there is a significant improvement $(p-$ value $=0.0467)$ between the results reached by the maximum aggregation and those obtained by using the mean operator.

The Friedman aligned rank test (see Figure 2) obtains a low p-value $(<0.0001)$ when comparing the four methods at the same time, which implies that there are significant differences between the results provided by each method. In the case of Canny (see Figure 3) we

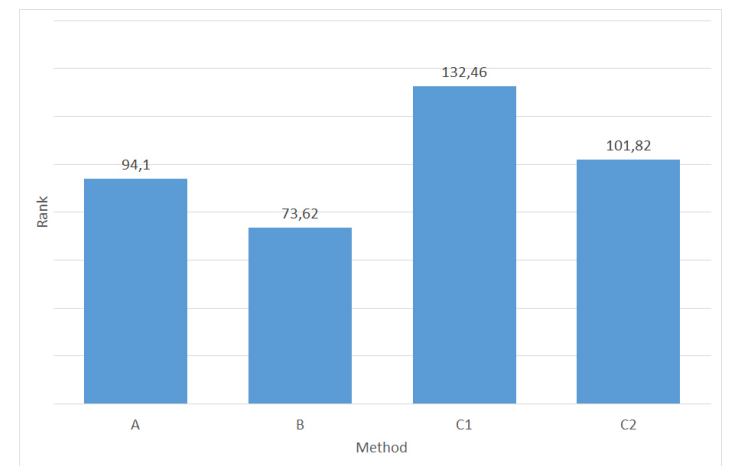

Figure 3: Rankings of the three compared methods employing Canny's algorithm.

find as well differences but this time is Method B best ranked compared with C1's and C2's, and Method A is better compared with C1's.

\begin{tabular}{ccc} 
Algorithm & Hypothesis & APV \\
\hline Method A & Rejected for Method C2 & $\mathbf{0}^{*}$ \\
Method B & Rejected for Method C2 & $\mathbf{0 *}^{*}$ \\
Method C1 & Not rejected for Method C2 & 0.5887 \\
\hline
\end{tabular}

Table 5: Holm test to compare Method C2 against the others.

After the Sobel's results we can apply a Holm test by using the best approach (the one with lower ranking) as control method and computing the adjusted p-value (APV) for the remaining three methods.

Table 5 clearly reflects the statistically significant differences between the control method (Method C2) and each one crispy based methods A or B. This is not enough evidence to reject the hypothesis of equivalence of both fuzzy approaches when considering a multiple comparison scheme.

\section{Conclusions}

As a remarkable novelty of this paper, we have clarified different methodologies for aggregating the color information of RGB images in the edge detection problem. Moreover, we have found differences in the quality of the comparatives depending of which methodology is applied. In the case of Canny's we could not find improvements for the soft approach as we did with Sobel's. This is due to the fact that the hysteresis is affected in a complex way by the aggregation function employed. We believe that this aspect deserves a deeper research, and this will show a connection between global evaluation approach (see [9]) and color edge detection.

Future research could point out the use of different 
thresholds for each channel. This seems a natural way to continue with this research. This is not been explored here as the number of possibilities and casuistics grows significatively, which leads towards a much longer paper. As well, more research is needed for working with new aggregations, i.e the weighted sum of the color channels. We think that this aggregation function could result in better comparatives against humans due to the slightly different weight of each color in human vision. As well, different aggregation functions could be applied depending of the edge detection algorithm employed. Finally, a more complex line for aggregating colors could be based on the use of other color space different from the RGB's.

\section{Acknowledgement}

This research has been partially supported by the Government of Spain, grant TIN2015-66471-P.

For the conducting of this research, the code created by Kermit Research Unit has been strongly helpful [3].

\section{References}

[1] S. Bogumil, Color image edge detection and segmentation: A comparison of the vector angle and the euclidean distance color similarity measures, Ph.D. thesis, University of Waterloo (1999).

[2] J. Canny, A computational approach to edge detection, IEEE Transactions on Pattern Analysis y Machine Intelligence PAMI-8 (6) (1986) 679-698.

[3] B. de Baets, C. López-Molina, The kermit image toolkit (kitt), Ghent university, www.kermitimagetoolkit.net.

[4] J. Demšar, Statistical comparisons of classifiers over multiple data sets, Journal of Machine learning research 7 (Jan) (2006) 1-30.

[5] S. Di Zenzo, A note on the gradient of a multiimage, Computer vision, graphics, and image processing 33 (1) (1986) 116-125.

[6] S. Dutta, A color edge detection algorithm in rgb color space, 2009, pp. 337-340.

[7] P. Flores-Vidal, N. Martínez, D. Gómez, Postprocessing in edge detection based on segments, in: Proceedings of the 13th International FLINS Conference on Data Science and Knowledge Engineering for Sensing Decision Support, World Scientific Proceedings Series on Computer Engineering and Information Science (Belfast), World Scientific, 2018, p. 11.

[8] P. A. Flores-Vidal, J. Montero, D. Gómez, G. Villarino, A new edge detection method based on global evaluation using supervised classification algorithms, International Journal of Computational Intelligence Systems 11 (1).

[9] P. A. Flores-Vidal, P. Olaso, D. Gómez, C. Guada, A new edge detection method based on global evaluation using fuzzy clustering, Soft Computing (2018) 1-13.

[10] S. García, A. Fernández, J. Luengo, F. Herrera, Advanced nonparametric tests for multiple comparisons in the design of experiments in computational intelligence and data mining: Experimental analysis of power, Information Sciences 180 (10) (2010) 2044-2064.

[11] C. Guada, D. Gómez, J. T. Rodríguez, J. Yáñez, J. Montero, Classifying image analysis techniques from their output, International Journal of Computational Intelligence Systems 9 (2016) 43-68, cited By :4.

[12] C. Guada, D. Gómez, J. T. Rodríguez, J. Yáñez, J. Montero, Fuzzy image segmentation based on the hierarchical divide y link clustering algorithm, in: Proceedings - The 2015 10th International Conference on Intelligent Systems y Knowledge Engineering, ISKE 2015, 2016, pp. 12-17.

[13] M. Heath, S. Sarkar, T. Sanocki, K. Bowyer, Comparison of edge detectors: A methodology and initial study, Computer Vision and Image Understanding 69 (1) (1998) 38-54.

[14] S. Holm, A simple sequentially rejective multiple test procedure, Scandinavian journal of statistics (1979) 65-70.

[15] N. P. Jacobson, M. R. Gupta, Design goals and solutions for display of hyperspectral images, IEEE Transactions on Geoscience and Remote Sensing 43 (11) (2005) 2684-2692.

[16] D. Marr, Vision: a Computational Investigation into the Human Representation y Processing of Visual Information, 1982.

[17] D. R. Martin, C. Fowlkes, D. Tal, J. Malik, A database of human segmented natural images y its application to evaluating segmentation algorithms y measuring ecological statistics, in: Proceedings of the IEEE International Conference on Computer Vision, Vol. 2, 2001, pp. 416-423.

[18] M. A. Ruzon, C. Tomasi, Color edge detection with the compass operator, in: Proceedings. 1999 IEEE Computer Society Conference on Computer Vision and Pattern Recognition (Cat. No PR00149), Vol. 2, 1999, pp. 160-166 Vol. 2. 
[19] I. Sobel, History and definition of the so-called "sobel operator", more appropriately named the sobel-feldman operator.

[20] P. E. Trahanias, A. N. Venetsanopoulos, Color edge detection using vector order statistics, IEEE Transactions on Image Processing 2 (2) (1993) 259-264.

[21] F. Wilcoxon, Individual comparisons by ranking methods, Biometrics bulletin 1 (6) (1945) 80-83.

[22] Y. Yang, Colour edge detection and segmentation using vector analysis, 1995. 